\title{
The diagnostic value of circulating microRNAs as a biomarker for gastric cancer: A meta-analysis
}

\author{
HUI WEI ${ }^{1,2^{*}}, \mathrm{KE} \mathrm{PU}^{1,2^{*}}$, XIAO-GUANG LIU ${ }^{1,2}$, BO-XUAN LI ${ }^{3,4}$, HENG-SHUO ZHANG ${ }^{5}$, \\ HUAN WANG ${ }^{5}$, HAO WANG ${ }^{6}$, WEI-MING SUN ${ }^{7}$ and YU-PING WANG ${ }^{1,2}$ \\ ${ }^{1}$ Department of Gastroenterology, The First Hospital of Lanzhou University; \\ ${ }^{2}$ Key Laboratory for Gastrointestinal Diseases of Gansu Province, Lanzhou University; \\ ${ }^{3}$ School of Pharmacy, Lanzhou University; ${ }^{4}$ Department of Pharmacy, The First Hospital of Lanzhou University; \\ ${ }^{5}$ The First Clinical Medical School of Lanzhou University; ${ }^{6}$ The Second Clinical Medical School of Lanzhou University; \\ ${ }^{7}$ Department of Endocrinology, The First Hospital of Lanzhou University, Lanzhou, Gansu 730000, P.R. China
}

Received March 16, 2018; Accepted October 8, 2018

DOI: $10.3892 /$ or.2018.6782

\begin{abstract}
Recently, cancer research microRNA studies have drawn great attention. However, the results of these studies have been inconsistent and variable regarding the availability of circulating miRNAs in gastric cancer (GC) diagnosis. Thus, results should be interpreted cautiously. The purpose of the present study was to assess the diagnostic performance of circulating miRNAs in GC diagnosis. We conducted a systematic and comprehensive approach for the inclusion of studies. The sensitivity, specificity, and diagnostic odds ratio were pooled with random effects models, and a summary of receiver operator characteristic (SROC) curves were plotted. The potential heterogeneity was assessed with $\mathrm{Q}$ test and $\mathrm{I}^{2}$ statistics. Subgroup analyses and meta-regressions further investigated the sources of heterogeneity. A total of 77 studies from 48 articles were eligible for the meta-analysis. The results revealed a sensitivity of 0.76 , a specificity of 0.81 , and an AUC of 0.86 for gastric cancer diagnosis with circulating miRNAs. In addition, subgroup analyses indicated that multiple miRNAs assays, non-microarray screening approaches, and serum-based miRNA assays exhibited good diagnostic performance in contrast to a single miRNA assay,
\end{abstract}

Correspondence to: Professor Yu-Ping Wang, Department of Gastroenterology, The First Hospital of Lanzhou University, Lanzhou, Gansu 730000, P.R. China

E-mail: wangyuping@lzu.edu.cn

*Contributed equally

Abbreviations: AUROC, area under the receiver operating characteristic curve; CI, confidence interval; DOR, diagnostic odds ratio; FN, false negative; FP, false positive; GC, gastric cancer; LR, likelihood ratio; QUADAS, quality assessment of diagnostic accuracy studies; SE, sensitivity; SP, specificity; TP, true positive; $\mathrm{TN}$, true negative; TNM, tumor-node-metastasis

Key words: microRNAs, GC, circulation, meta-analysis, biomarker microarray expression profiling screening, and plasma-based miRNA group analysis. The diagnostic ability of miRNAs in early stage I-II groups and the high expression group were approximately similar to that in the stage I-IV groups and the low expression group. For the circulating miRNAs, our meta-analysis identified a combination of multiple miRNAs, non-microarray chip screening, and serum-based miRNA assays were associated with the most effective GC diagnostic performance. However, many unclear molecular mechanisms limited the accuracy of the diagnostic results, and should be interpreted with caution. Further large-scale prospective studies are required for validating the diagnostic applicability of circulating miRNAs in gastric cancer patients.

\section{Introduction}

Gastric cancer (GC) is the sixth most common cancer and the fourth leading cause of cancer-related deaths worldwide (1-3). The incidence of GC, especially in the Asian region (China, Japan, and Korea), has markedly increased over the past three decades (4). Moreover, statistics of cancer research from the International Agency for Research on Cancer have revealed that the morbidity and mortality rates associated with GC in China accounted for 42.6 and $45.0 \%$ of the global rates in 2015, respectively (5). Consequently, the high incidence of GC can result in great health and economic burdens (6). The recent development of diagnostic and surgical techniques has greatly improved the prognosis of GC patients $(7,8)$. Five-year survival rates for patients with early GC can reach $90 \%$. Unfortunately, most patients, however, have already developed advanced GC at the time of diagnosis $(9,10)$. For patients with advanced GC, several studies have revealed that the median survival time of patients with GC was only 6-9 months, with surgical treatment unable to prolong the patient survival $(11,12)$. Even with expanded resection, lymph node clearance, and neoadjuvant chemotherapy, the incidence rates of postoperative local recurrence and distant metastasis are still high (13). Therefore, considering the high ratio of early GC patients who are cured, the key to reducing mortality rates and improving the prognosis for GC patients is early and accurate diagnosis. 
To create a non-invasive, convenient, and low-priced diagnostic method, circulating biomarker detection methods have been widely used in clinics. GC serum tumor markers are currently used in clinical settings for the detection of GC. However, methods for the detection of carcinoembryonic antigen (CEA), carbohydrate antigen 199 (CA199), and carbohydrate antigen 724 (CA724) lack adequate sensitivity and specificity, which has precluded their widespread application in early diagnosis of GC (14). Therefore, it is necessary to investigate other potential biomarkers useful in identifying GC with high sensitivity and specificity. Recently, many researchers have been attracted to microRNAs (miRNAs) which have been stably detected in cell-free body fluids, such as plasma or serum. These miRNAs are called circulating miRNAs and are protected from degradation by ribonucleases in the blood. Additionally, the expression profile of miRNAs in GC patients usually exhibits exceptionally high expression of miRNAs in contrast to that in normal specimens (15).

miRNAs are a small, single-stranded non-coding RNAs that can regulate the expression of thousands of genes by inhibiting and degrading mRNAs during the translation process $(16,17)$. miRNAs regulate various pathophysiological processes that involve cell proliferation, apoptosis, and differentiation. They also play an important role in tumorigenesis, especially neoplasm development, metastasis, angiogenesis, and immune responses (18).

Circulating miRNAs are considered to be novel potential biomarkers in the detection of many diseases, which were first demonstrated by Mitchell et al (19) for the detection of cancer. Subsequently, their roles have been demonstrated in numerous studies. Nevertheless, it is difficult to specify a suitable miRNA, and the results of miRNA expression analysis are inconsistent. Despite a large number of studies on circulating miRNAs, few relevant measures have been applied in the clinical setting. In the present study, in this meta-analysis, we systematically and comprehensively analyzed the diagnostic accuracy of circulating miRNAs in distinguishing gastric cancer patients from the significant heterogeneity of other factors.

\section{Materials and methods}

Literature search. Two authors independently searched online databases, PubMed, Embase, the Cochrane Library, Web of Science, and Springer to identify potentially eligible studies published before November 10, 2017. The keywords used for literature retrieval were ('microRNA' or 'miRNA' or 'miR') and ('gastric cancer' or 'gastric carcinoma' or 'gastric tumor' or 'gastric neoplasm') and ('diagnostic' or 'diagnosis' or 'sensitivity and specificity' or 'ROC curve') and ('circulating' or 'plasma' or 'serum' or 'blood'). The citations in identified articles and in review articles were also examined. All publications identified by our search strategy were independently assessed by four reviewers. Any disagreement on a controversial study was resolved by discussion to consensus.

Literature selection. Eligible studies included in the present meta-analysis met the following criteria: i) the diagnosis of GC was made based on histopathological confirmation; ii) miRNA concentration in plasma, serum, or blood was detected before the patient received any treatment; iii) the study explored the correlation between miRNA expression levels and gastric cancer diagnosis; iv) the study included standard references for the GC diagnosis, including patients with benign diseases or healthy individuals as the control groups; and v) the study provided adequate or sufficient data for the calculation of $2 \times 2$ tables consisting of true positive (TP), false positive (FP), true negative (TN), and false negative (FN). In addition, exclusion criteria were: i) publications irrelevant to the detection values of circulating miRNAs for GCs; ii) review studies, editorials, case reports, and letters; iii) duplicate publications; and iv) unqualified data. All literature that satisfied the above criteria constituted qualified studies.

Data extraction and quality assessment. The following data characteristics were extracted for each eligible study by two reviewers (HW and KP), independently: i) basic characteristics of the included studies, including the first author, year of publication, country of publication, origin of the study population, sample type, study design, sample size, number of participants, and variables adjusted for the analysis, and ii) for diagnostic studies, the sensitivity, specificity, TP, FP, FN, and $\mathrm{TN}$ values of the diagnostic test results were considered.

The quality assessment of the studies included in the present study was performed by two authors independently using the Quality Assessment of Diagnostic Accuracy studies (QUADAS-2) (20) checklist in Rev Man 5.0 software (http://ims.cochrane.org/revman/download). This scale was composed of four domains consisting of patient selection, index test, reference standard, and flow and timing domain. Each signaling question was judged as 'yes', 'no', or 'unclear' and each study's risk of bias and concern for applicability was estimated as 'high', 'low', or 'unclear' except for the flow and timing domain, for which the applicability concern did not apply. An answer of 'yes' meant that the risk of bias could be judged as being low, whereas an answer of 'no' or 'unclear' meant that the risk of bias could be judged as being high. A third reviewer was consulted in the case of conflict and inconsistency was dealt with by a multilateral argument.

Statistical methods. To evaluate the diagnostic accuracy of miRNA assays for detecting GC, the sensitivity and specificity parameters were extracted from each study and the number of patients with TP, FP, TN, and FN results from each included article were extracted or calculated. Based on the $2 \times 2$ tables, meta-analyses were performed with Meta-Disc software version 1.4 (http://www.hrc.es/investigacion/metadisc_en .htm.) and STATA software version 12.0 (Stata Corporation, College Station, TX, USA) to evaluate the pooled statistics (95\% CI) of sensitivity, specificity, positive and negative likelihood ratios (PLR and NLR) [PLR = sensitivity/(1 - specificity $),$ NLR = (1 - sensitivity $) /$ specificity], diagnostic odds ratio (DOR), and area under the summary receiver operating characteristic curves (AUSROC), with standard errors (SE) and $\mathrm{Q}$ index with SE for the test's performance using miRNA for GC detection. If sufficient information was not available, we recalculated these values on the basis of the sensitivity and specificity offered. Summary statistics revealed the diagnostic threshold effects that were analyzed by Spearman's correlation coefficient and P-value. If there was no significant threshold effect, the diagnostic accuracy was estimated by pooled 


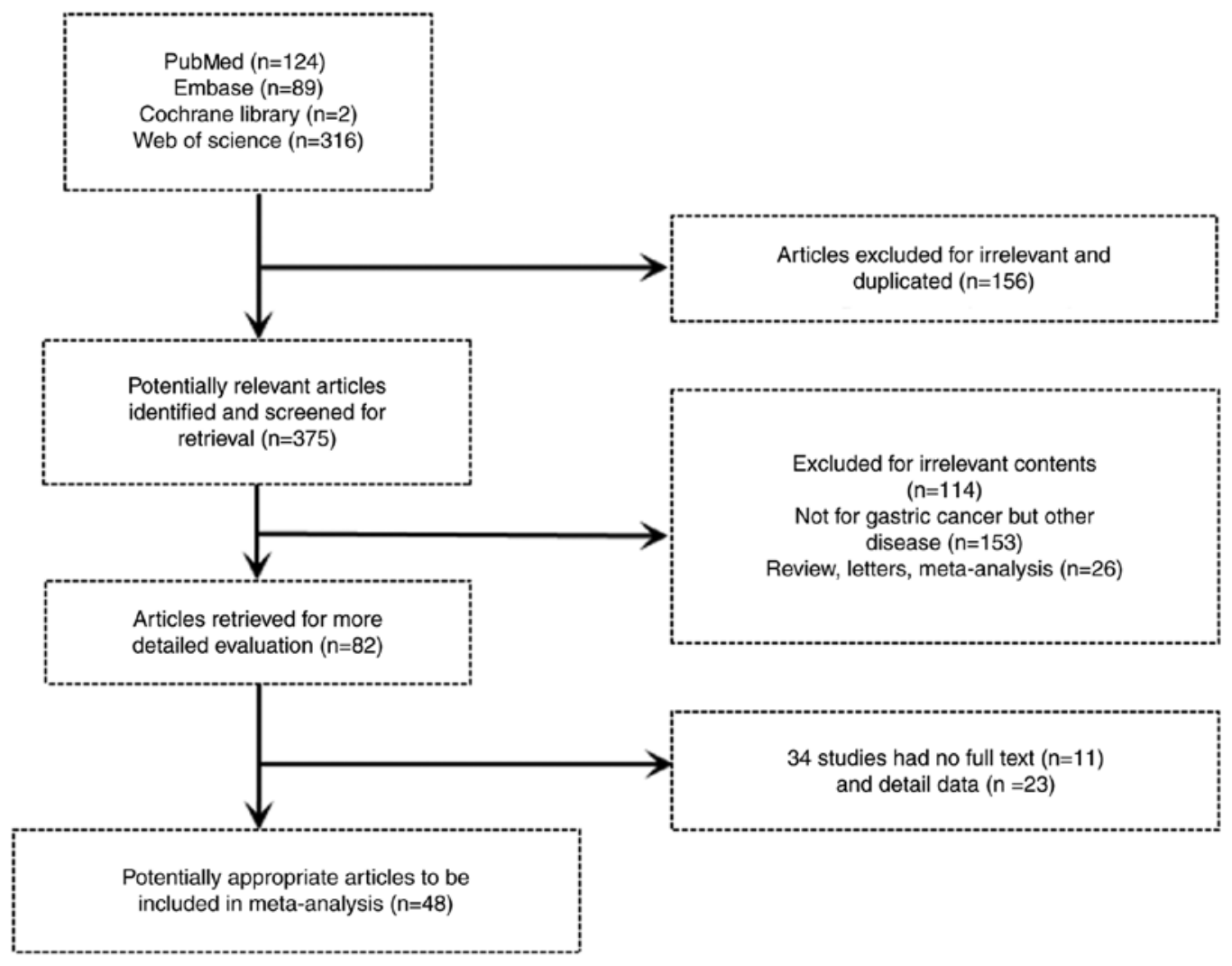

Figure 1. Flowchart for the selection of included articles is presented.

statistics, whereas the diagnostic accuracy was evaluated only by AUSROC and Q indices rather than sensitivities, specificities, PLR, NLR, and DOR.

Positive and negative likelihood ratios describe the discriminatory properties of positive and negative test results, respectively (21). Likelihood ratios state how many times more likely particular test results are in patients with disease than in those without disease (22). Positive likelihood ratios above 10 and negative likelihood ratios below 0.1 have been noted as providing convincing diagnostic evidence, whereas PLR $>5.0$ and NLR $<0.2$ imply higher diagnostic evidence (23). DOR represented the positive odds of aberrant miRNA expression in patients with GC compared to the probability of the healthy control. AUSROC values of 0.5-0.7, 0.7-0.9, and 0.9-1.0 were applied to indicate low, moderate, and high diagnostic accuracy, respectively. A smaller Q index indicated a lower diagnostic accuracy.

Heterogeneity was determined by Cochran's Q statistic based on the Chi-square test and $\mathrm{I}^{2}$ statistics. $\mathrm{I}^{2}$ values of $0-40$, 40-70, and 70-100\% indicated low, moderate, and high variance, respectively (24). If moderate heterogeneity existed or different clinical characteristics were noted, the DerSimonian and Laird random-effects model was applied. Considerable heterogeneity was considered if $\mathrm{I}^{2}>50 \%$ and/or $\mathrm{P}<0.05(25,26)$. Sources of heterogeneity were explored by meta-regression analysis based on possible characteristics and a subsequent subgroup analysis was performed to identify potential covariates. Furthermore, subgroup and meta-regression analyses were performed to detect the extent of heterogeneity between studies. Publication bias was checked using Deeks' funnel plot analysis (24). All of the aforementioned statistical calculations were made with Meta-DiSc and STATA 12.0 software.

\section{Results}

Literature search. In Fig. 1, the flowchart for the selection of included articles is presented. Searching Pubmed, Embase, the Cochrane library, and Web of Science resulted in the inclusion of 531 articles. After a review of titles and abstracts, 156 publications were found to be irrelevant or duplicated. Next, we, intensively read the remaining studies, whereby 114 of these publications were removed for irrelevant content, 153 articles were eliminated owing to the study being unrelated to gastric cancer, and 26 studies were not considered as they were review letters and meta-analyses. After a more detailed evaluation, 34 studies were removed as they did not contain full text or had insufficient data for extraction. Finally, the selection process revealed 48 studies that were eligible for diagnostic analysis.

Study characteristics and quality assessment. The main characteristics of the 48 qualified articles included 77 microRNAs, of which one study was performed in Europe and 47 studies were undertaken in Asia. The evaluated studies included a total of 3,829 cases and 3,175 controls for the present meta-analysis. These are presented in Table I. In the tumor-node-metastasis (TNM) classification, 20 articles included patients in stages I-IV. The other 13 included patients in stages I-II, of which only one study involved patients in stage I, whereas the remainder of the eligible studies $(n=15)$ did not mention the TNM stage. Analysis of data from the nine included 


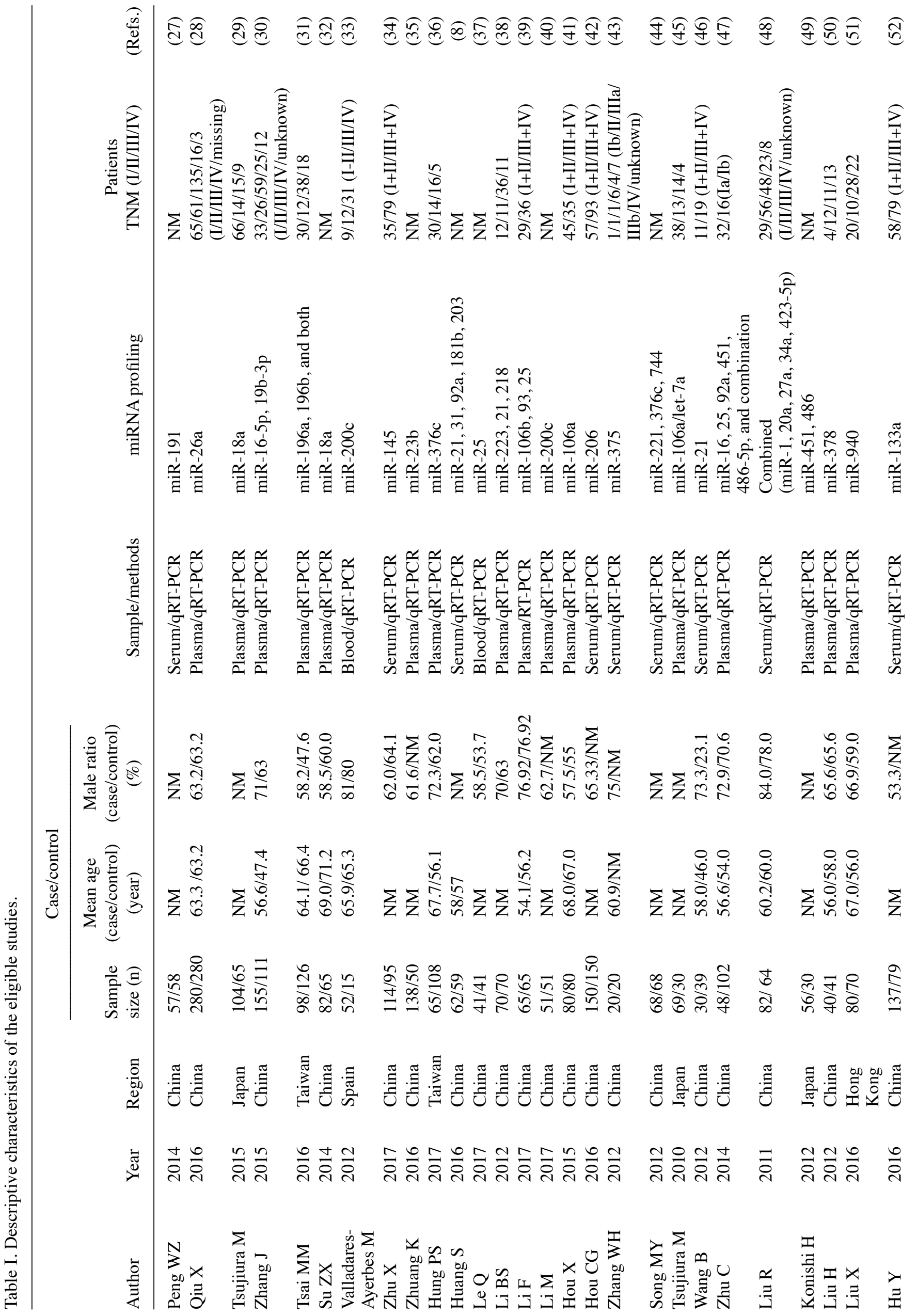




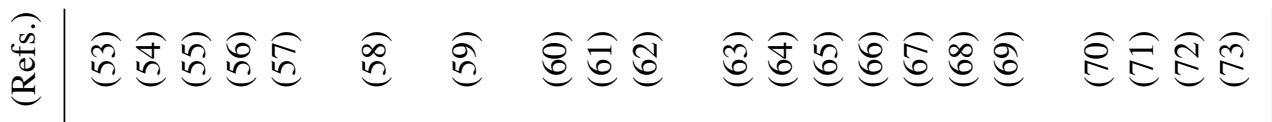

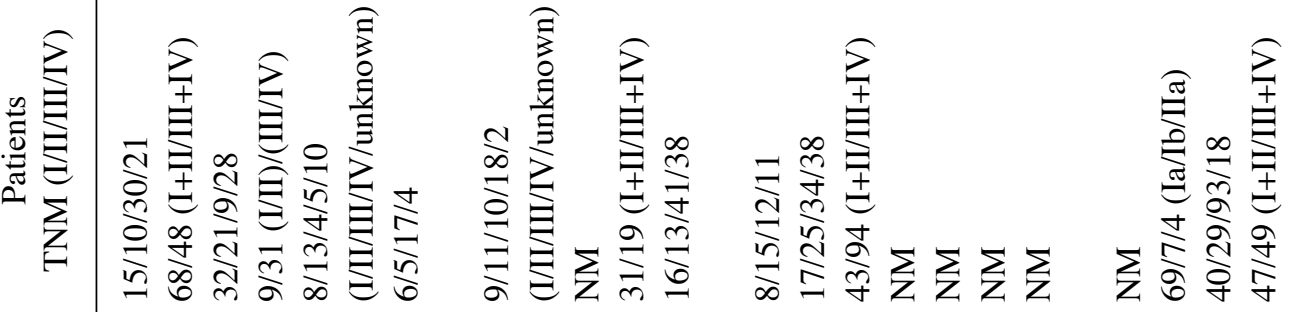

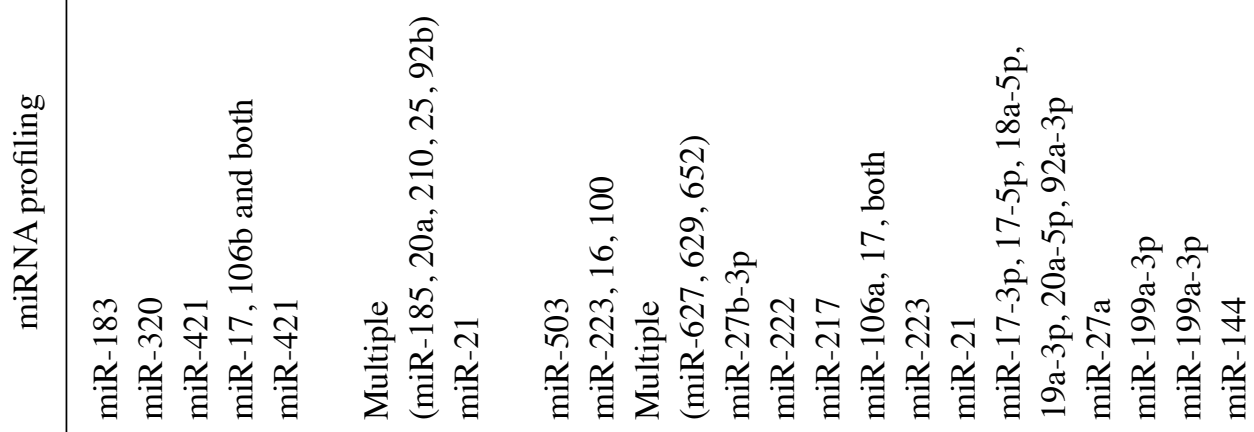

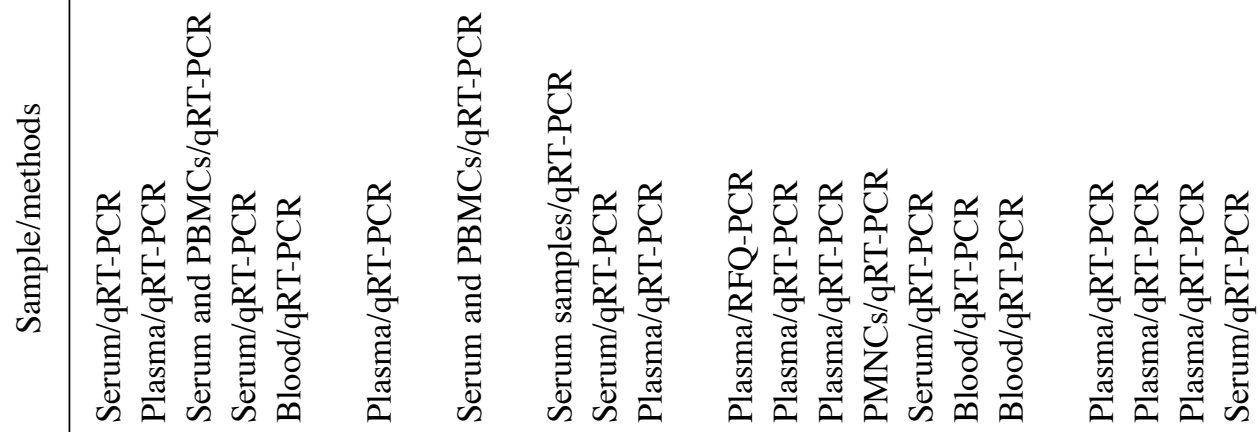

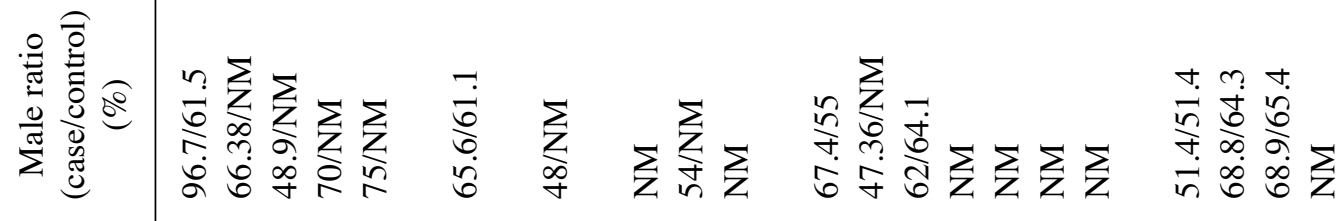

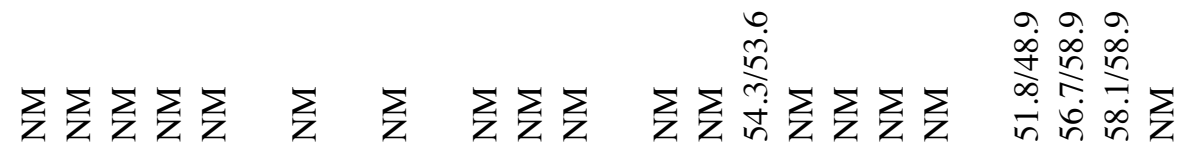

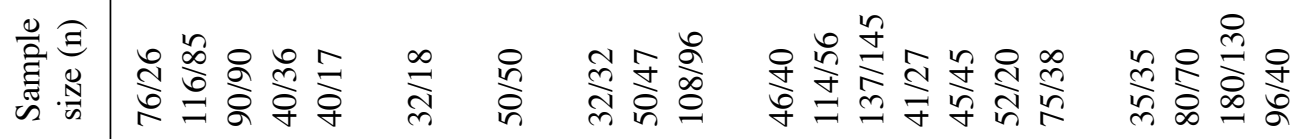

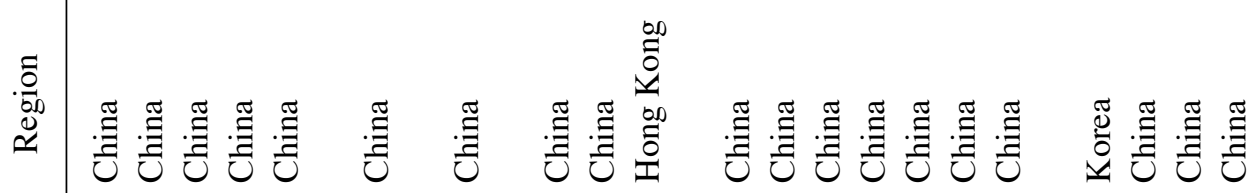

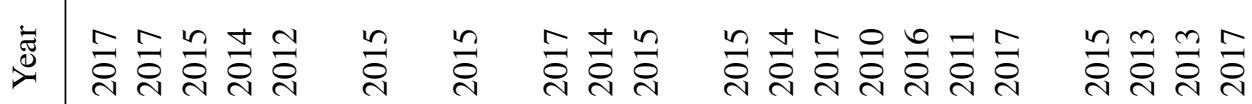

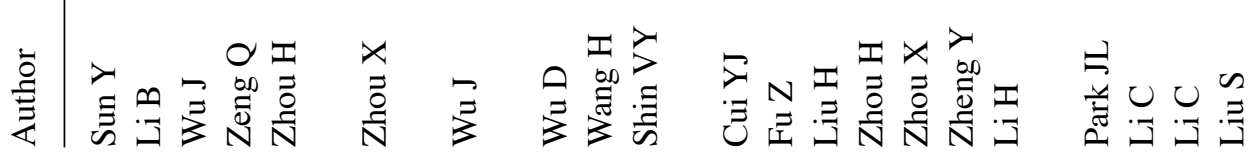




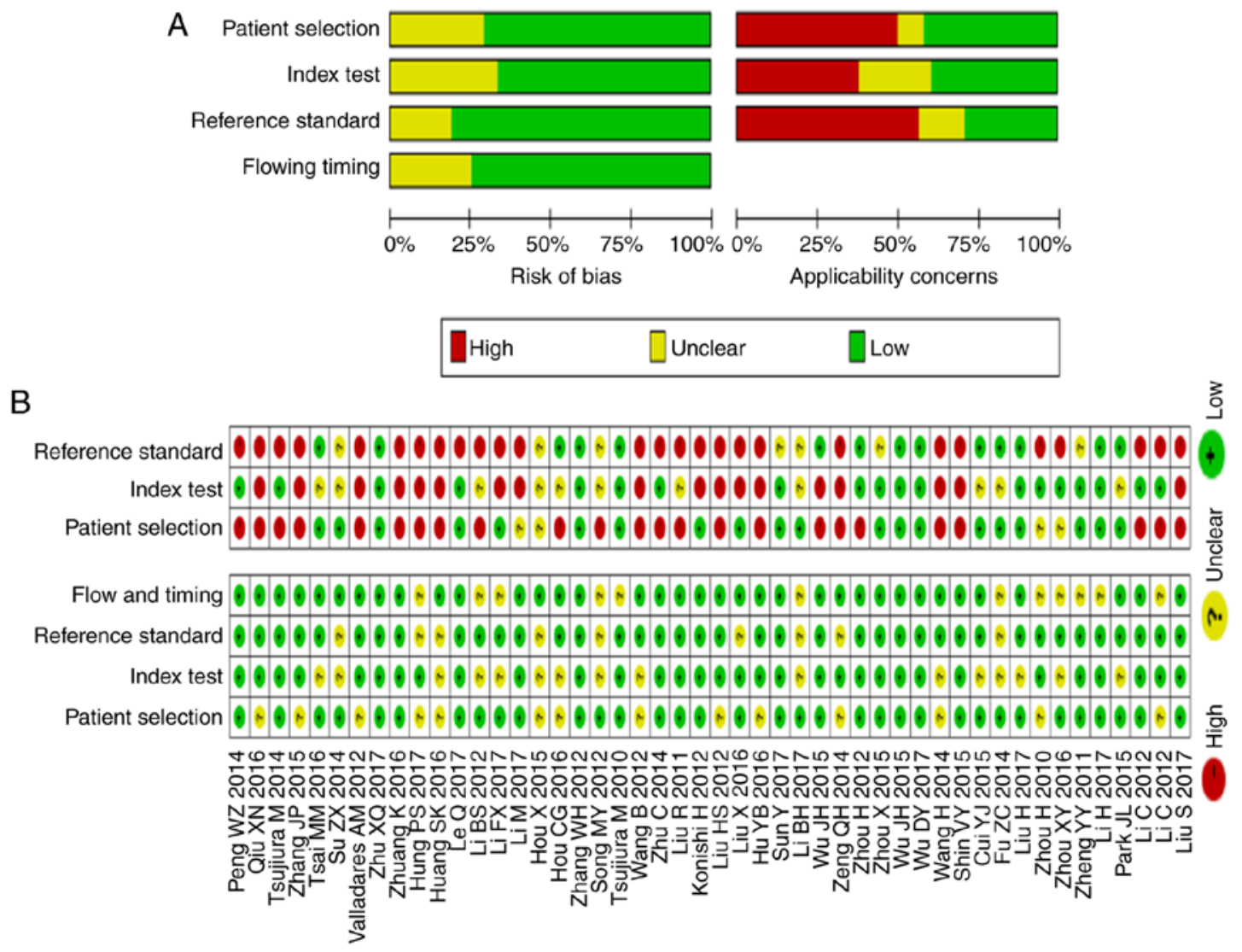

Figure 2. Assessment of the quality of the included studies using (A) a methodological quality graph and (B) the Cochrane Handbook.

studies that used miRNA microarray chips and revealed a number of miRNAs with altered expression, where candidate miRNAs were chosen via the training and validation design, whereas candidate miRNAs from the remaining articles $(n=39)$ were chosen directly without microchip procession. Meanwhile, 42 of the 48 studies investigated the diagnostic value of a single miRNA used in GC detection, while only nine researched a set of miRNAs. Of the selected miRNAs, three were from the single miRNA studies. In terms of samples, circulating miRNAs from GC and healthy individuals were classified as serum $(n=17)$, plasma $(n=25)$, and peripheral blood $(n=6)$. We also summarized miRNAs whose expression was upregulated in 33 studies and downregulated in 15 studies. One study (8) was excluded due to the unclear description about miRNA regulation, while another study (38) involved both upreguated and downregulated miRNAs. In particular, the expression of 58 miRNAs, including miR-21, was most frequently upregulated and that of 18 miRNAs (miR-26a and miR-199) was downregulated.

The quality of the eligible studies which were assessed, based on the QUADAS-2 criteria, was independently appraised by reviewers and is reported in Fig. $2 \mathrm{~A}$ and $\mathrm{B}$. In total, only five studies were valued as being low risk for bias and applicability concerns. The remaining studies were estimated as suboptimal for unclear risk in areas including index test, reference standard, flow, and timing. Most of the studies were identified as having a potential bias risk for patient selection and reference standard.

Diagnostic accuracy of circulating miRNA in GC. First, Meta-DiSc software version 1.4 was used to analyze the data.
The heterogeneity test found that $\mathrm{Q}$ test and $\mathrm{I}^{2}$ values of DOR were 233.49 and $67.5 \%$, respectively $(\mathrm{P}=0.0000)$ (Fig. 3A). Next, we generated forest plots of sensitivity and specificity, both of which did not display a straight line distribution and the Cochran-Q values were 369.52 and 555.63 (Fig. 3B and C), respectively, which reflected substantial heterogeneity (79.4 and $86.3 \%$, respectively) among these studies. Random-effects models were then selected to re-analyze the data and the diagnostic threshold was analyzed. The Spearman's correlation coefficient was $0.253(\mathrm{P}=0.026)$, illustrating that the significant heterogeneity was partially caused by the diagnostic threshold. In addition, this may be caused by discrepancies in the study approaches, specimen type, endogenous reference, or total sample size. Thus, we could not calculate the statistical outcomes indirectly by neglecting the different factors and owing to the high heterogeneity. The data could not simply be pooled and was only suitable for subgroup analyses for illustrating heterogeneity.

Covariates and subgroup analysis. After stratification in accordance with the five pre-specified covariates, including patients' stage of GC (early TNM stage I-II vs. all TNM stages I-IV), miRNA profiling (single miRNA vs. multiple miRNAs), specimen types (plasma vs. serum vs. blood), miRNA screening approaches (microarray processing vs non-microarray processing), and aberrant expression (upregulation vs. down-regulation). we next assessed their impact on sensitivity or/and specificity as shown in Table II and Fig. 4. Comparing different TNM stages of GC patients with altered expression of circulating miRNAs, the results revealed that 


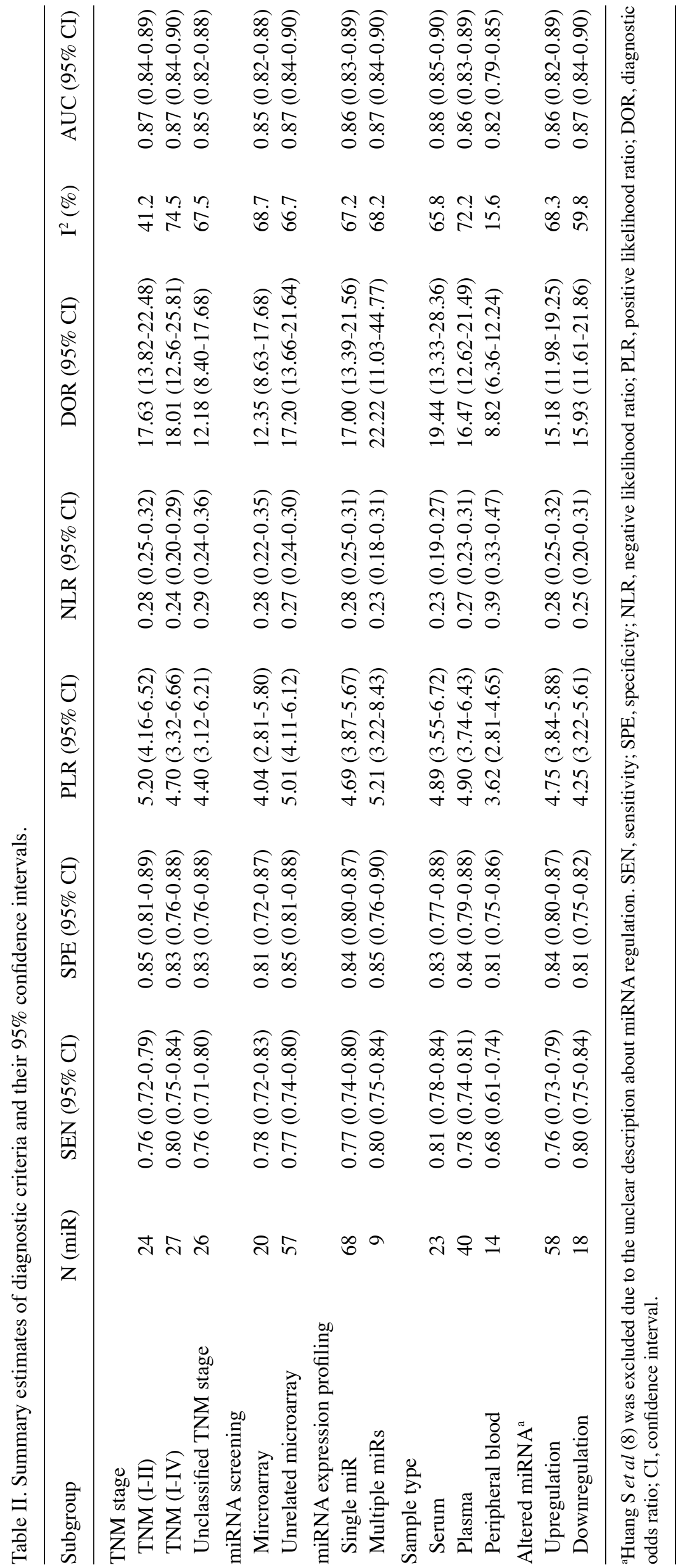



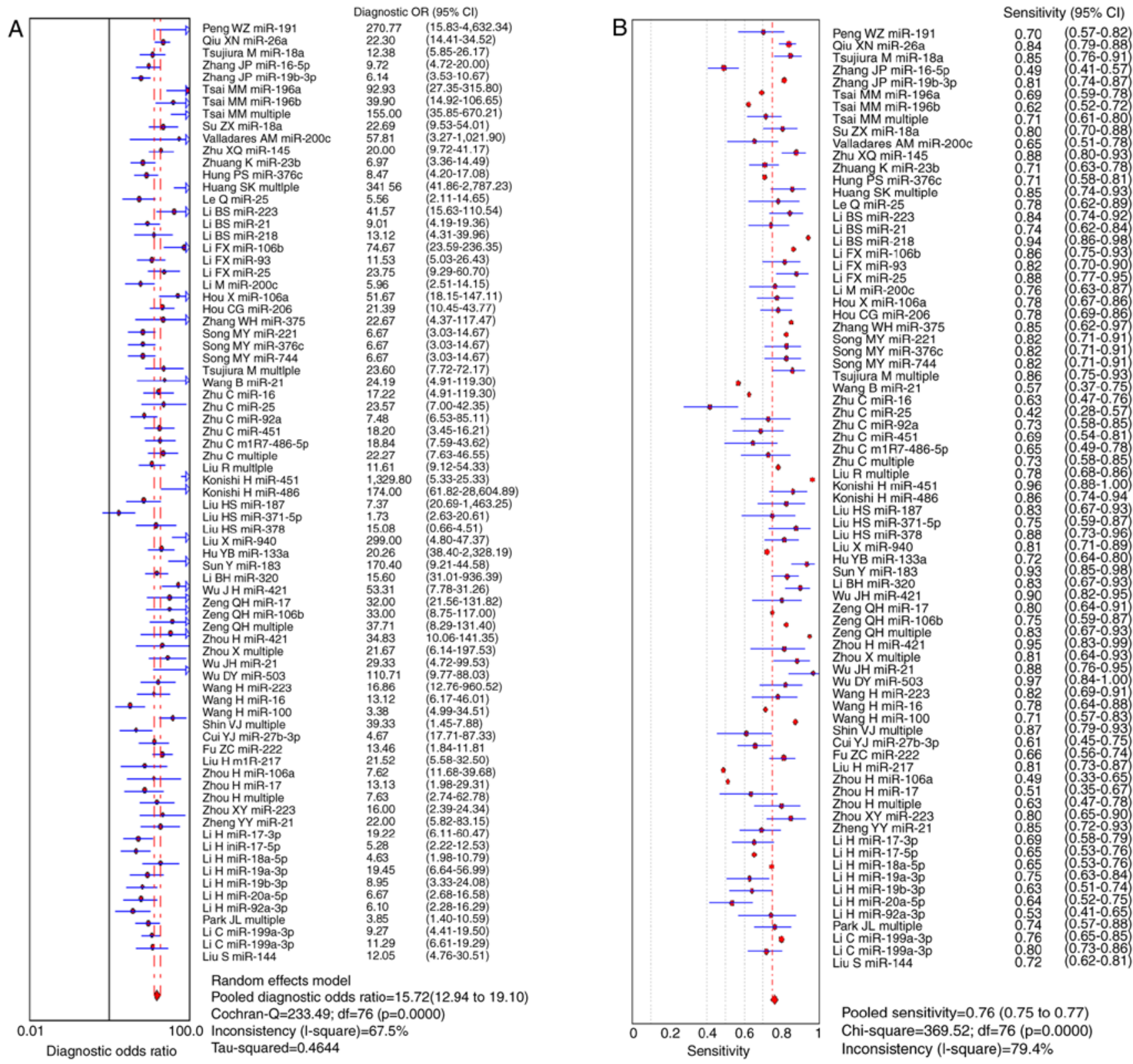

Figure 3. (A) Pooled diagnostic odds ratio (DOR) of circulating miRNAs in the diagnosis of gastric cancer patients. Forest plots and meta-analyses of studies showing the pooled (B) sensitivity of circulating miRNAs for diagnosing gastric cancer patients.

the diagnostic accuracy of miRNA detection during early stages I-II (sensitivity, 0.76; specificity, 0.85; PLR, 5.20; NLR, 0.28; DOR, 17.63; and AUC, 0.87) was similar to that during all stages I-IV (sensitivity, 0.80; specificity, 0.83; PLR, 4.70; NLR, 0.24; DOR, 18.01; and AUC, 0.87) and non-description stages (sensitivity, 0.76; specificity, 0.83; PLR, 4.40; NLR, 0.29; DOR, 12.18; and AUC, 0.85) with respect to all parameters except for the slight disparity in DOR. These results indicated that these biomarkers could not differentiate early GC from other later TNM stages in accordance with the diagnostic value.

Subsequently, we focused on the different screening approaches, such as microarray processing vs. non-microarray processing, and applied these approaches to candidate miRNAs. The differences between the pooled estimates of DOR (Table II) between miRNAs originating from microarray and miRNAs selected directly implied that the diagnostic ability of the former was inferior to the latter. The AUSROC (Fig. 5) indicated that the diagnostic accuracy of miRNAs in microarray screening was slightly less than the non-microarray selection group. In addition, we conducted subgroup analyses based on miRNA profiling, including single miRNA and multiple miRNAs. In the subgroup analysis (Table II and Fig. 6), compared to that of single miRNA, the diagnostic ability of multiple miRNAs was better, with the sensitivity increasing from 0.77 to 0.80 and the specificity increasing from 0.84 to 0.85 . AUC varied from 0.86 to 0.87 and the DOR value markedly increased from 17.00 to 22.22 , whereas AUROC implied that there were parallel diagnostic accuracies between both.

Subgroup analysis of specimen types and aberrant expression were conducted to identify whether the candidate miRNAs could clearly discriminate GC sufficient diagnostic performance and accuracy. In the specimen type subgroup 


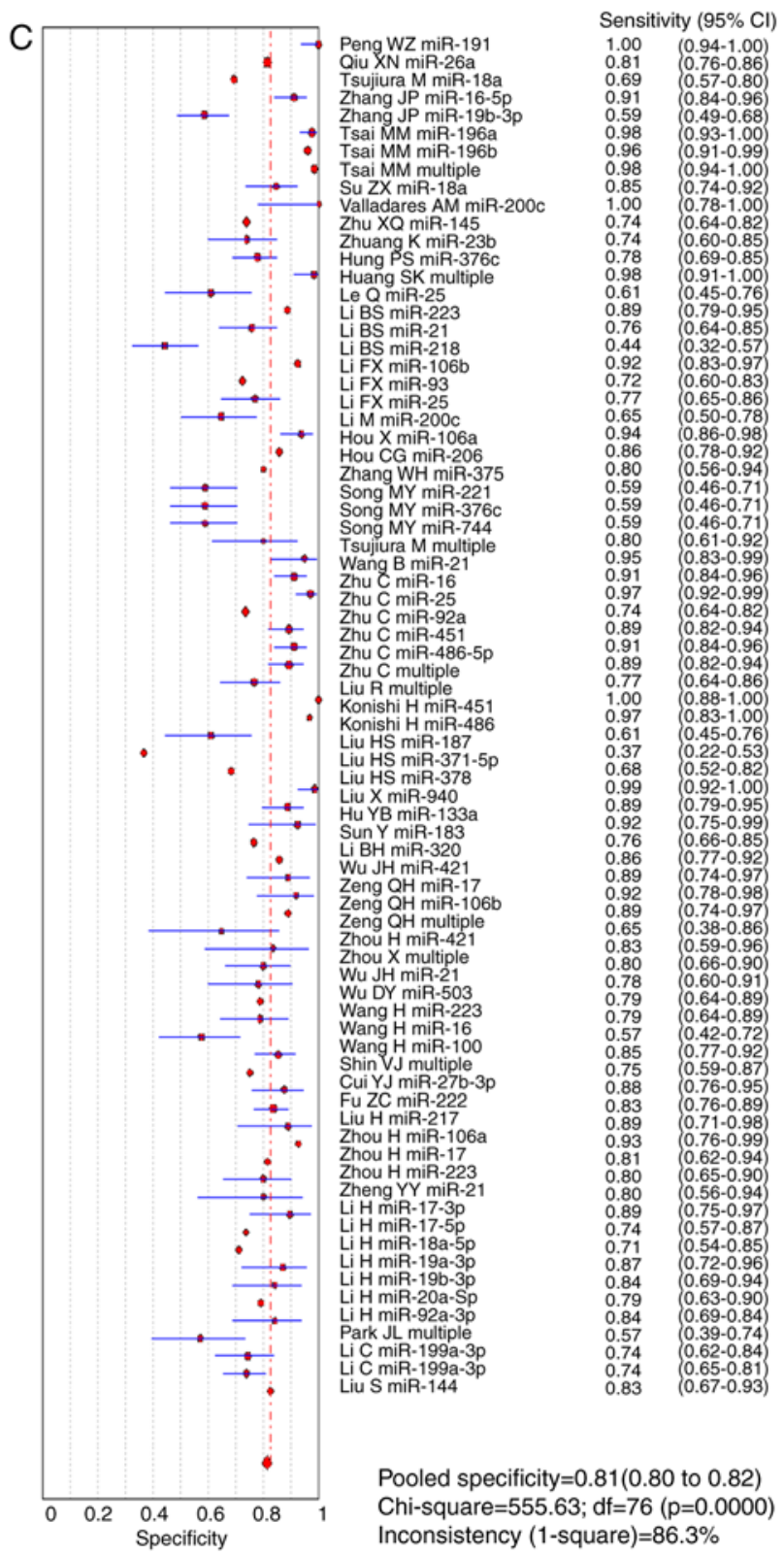

Figure 3. Continued. (C) Specificity of circulating miRNAs for diagnosing gastric cancer patients. revealed in Table II and Fig. 7, the diagnostic sensitivity of miRNAs extracted from serum was 0.81 and the specificity was 0.83 , with a pooled DOR of 19.44 and AUC of 0.88 . The sensitivity and specificity of miRNAs from plasma-based studies were 0.78 and 0.84 , respectively, with a summary DOR of 16.47 and AUC of 0.86 . However, for the peripheral blood assay, sensitivity and specificity were 0.68 and 0.81 , respectively, with a pooled DOR of 8.82 and AUC of 0.82, which indicated that the serum-based miRNA detection had a higher diagnostic value for GC than either the plasma or the peripheral blood-based assays. Moreover, further research was conducted to identify whether the upregulated miRNAs had better diagnostic accuracy than the downregulated miRNAs. Thus, the altered expression subgroup analyses for all miRNAs are shown in Table II and Fig. 8, the variable value in both changed slightly, according to the data of DOR (15.18 and 15.93) and AUC (0.86 and 0.87), which revealed that the diagnostic performance of miRNAs for GC detection in the high expression group was similar to that in the low expression group. From the above subgroup analysis that sought to determine the source of heterogeneity, the results indicated that the subgroups involving patients in the TNM (I-II) stage $\left(I^{2}=41.2 \%\right)$ using peripheral blood samples $\left(I^{2}=15.6 \%\right)$, contributed to moderate and mild heterogeneity, respectively. In addition, the decreasing trend in alteration implicitly suggested that the two factors may possibly be the source of heterogeneity. Nevertheless, further steps were taken to determine whether the aforementioned controversy led to the heterogeneity partly or entirely, which was confirmed by meta-regression analysis.

Meta-regression analysis. Based on the characteristics of the included studies, covariates, including TNM classification of GC, miRNA profiling, specimen types, miRNAs screening approaches, and aberrant expression status were applied to investigate inter-study heterogeneity using a meta-regression model shown in Table III (A-E). In the meta-regression analysis, sources of significant heterogeneity statistically indicated that the specimen type of the miRNA contributed significantly $(\mathrm{P}=0.0014)$, while the heterogeneity of results was not significantly influenced by the rest of the covariates. In accordance with the above
A

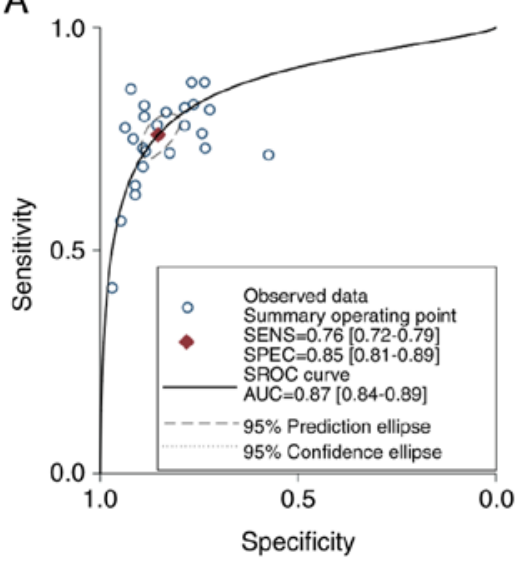

B

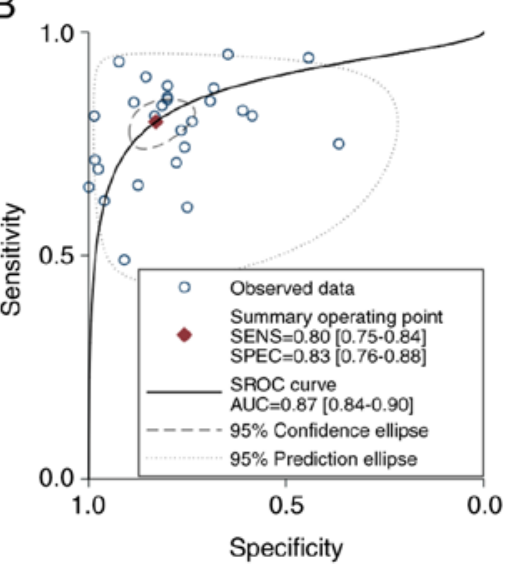

C

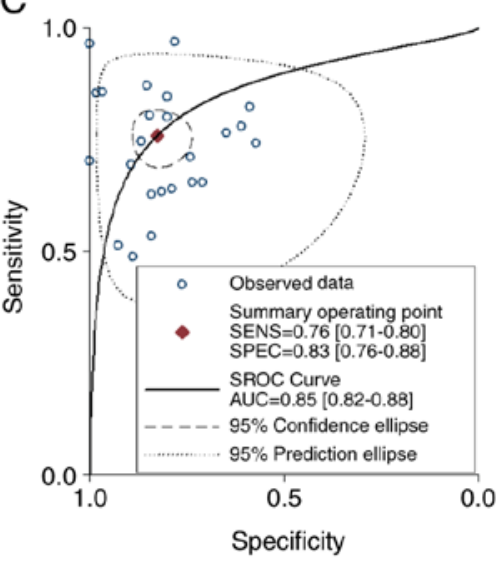

Figure 4. Summary of AUROC of circulating miRNAs from (A) early TNM stages (I-II), (B) TNM stages (I-IV), and (C) non-mentioned TNM stages for the diagnosis of gastric cancer patients. 

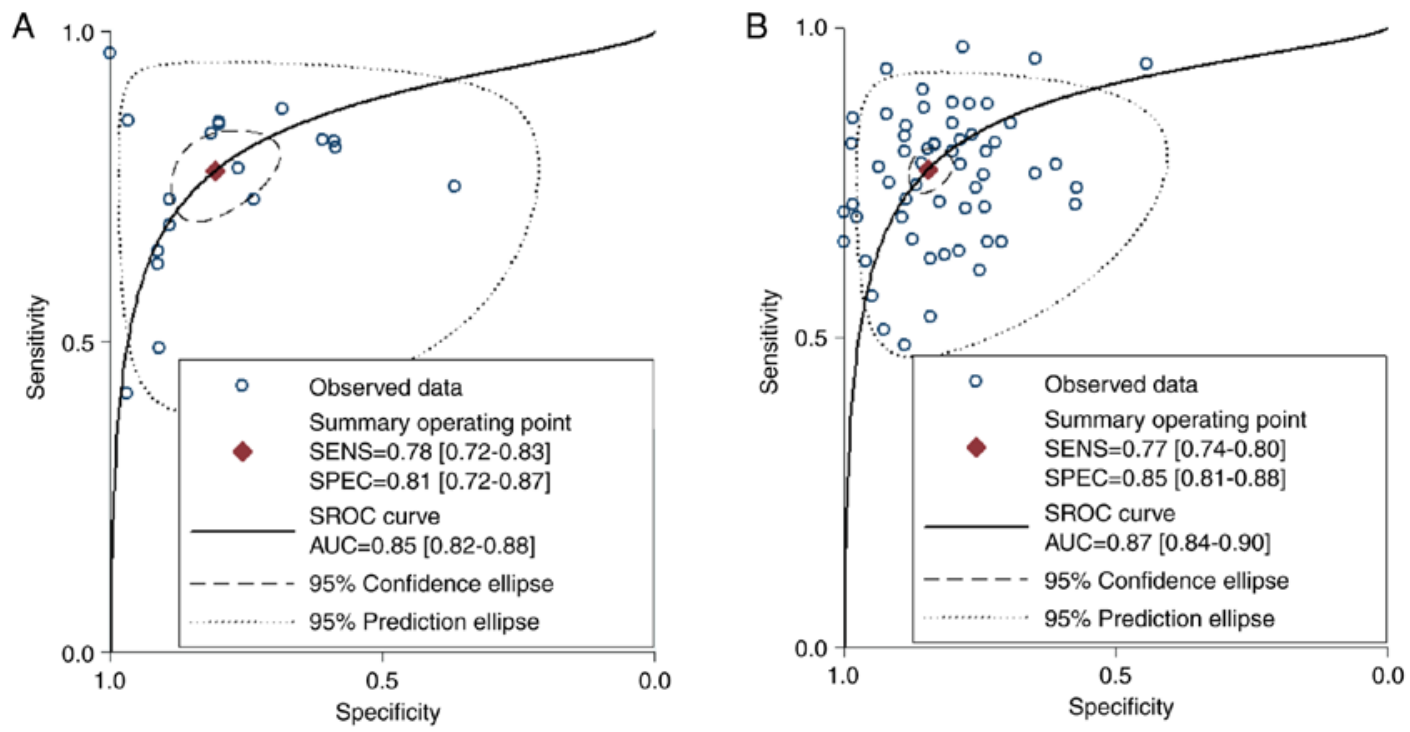

Figure 5. Summary of AUROC of circulating miRNAs from (A) microarray screening subgroup and (B) non-microarray screening subgroup for the diagnosis of gastric cancer patients.
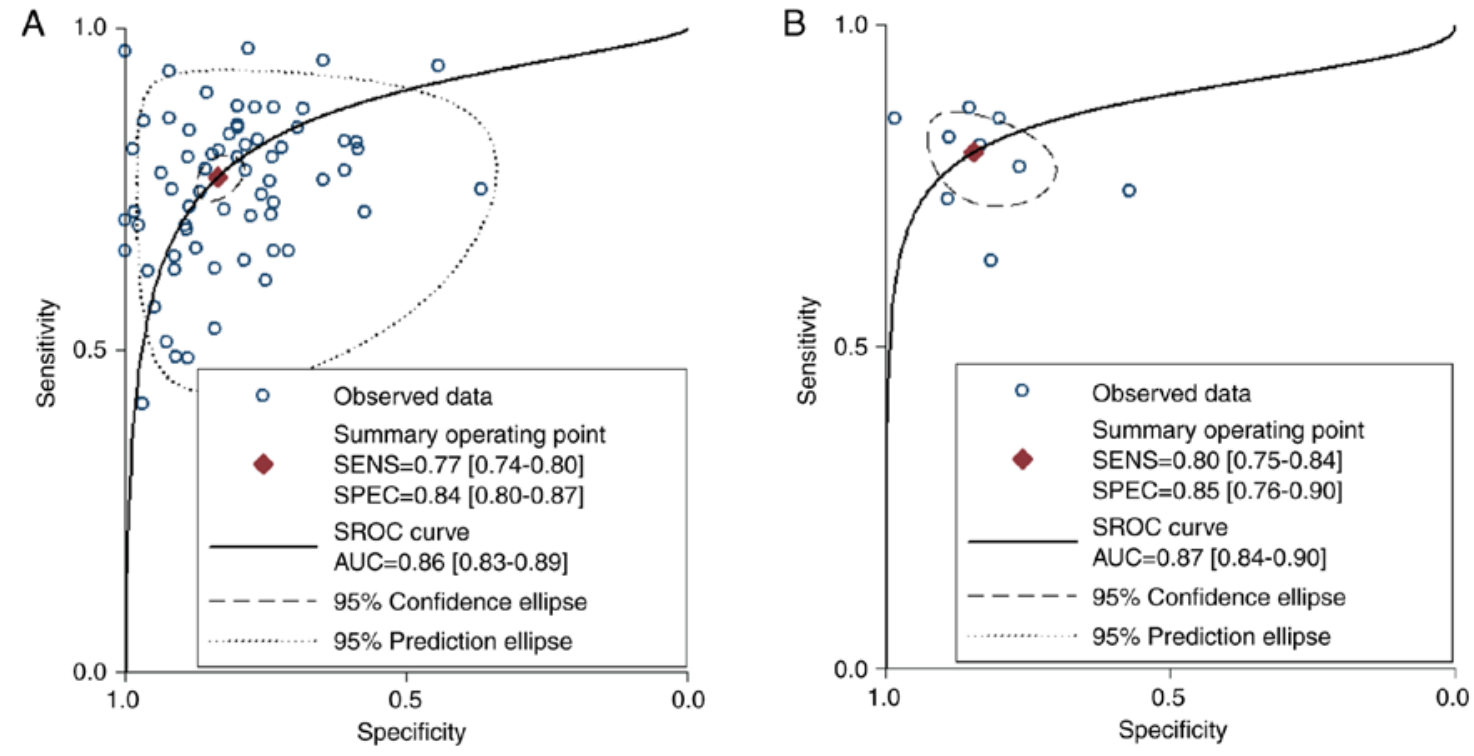

Figure 6. Summary of AUROC of circulating miRNAs from (A) a single miRNA and (B) multiple miRNAs for the diagnosis of gastric cancer patients.
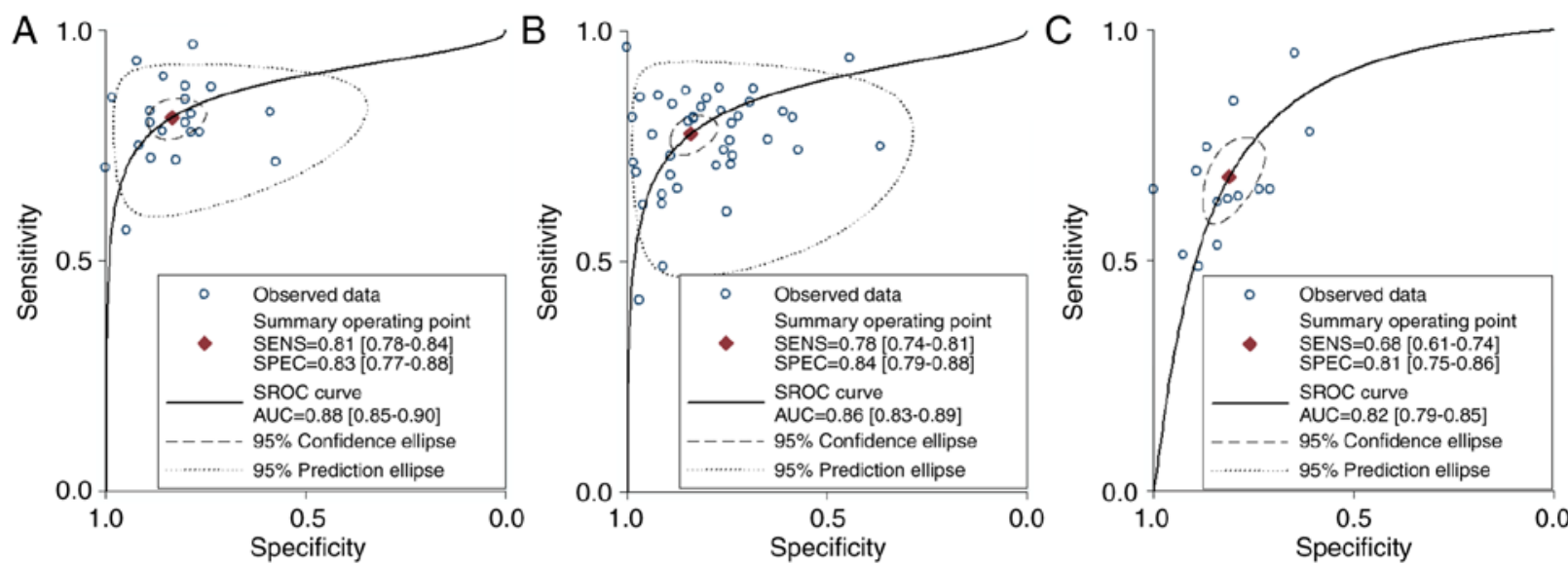

Figure 7. Summary of AUROC of circulating miRNAs from (A) serum-based specimens, (B) plasma-based specimens, and (C) peripheral blood-based specimens for the diagnosis of gastric cancer patients. 
Table III. Meta regression to evaluate the inter-subgroup heterogeneity of miRNAs for the diagnosis of gastric cancer patients.

A, Five covariates

\begin{tabular}{|c|c|c|c|c|c|}
\hline Variables & Coeff. & Std. Err & P-value & RDOR & $(95 \% \mathrm{CI})$ \\
\hline Cte. & 3.059 & 0.4343 & 0.0000 & - & - \\
\hline S & -0.301 & 0.0735 & 0.0001 & - & - \\
\hline TNM stage & 0.186 & 0.1119 & 0.1004 & 1.20 & $(0.96 ; 1.51)$ \\
\hline Up/downregulation & -0.012 & 0.2047 & 0.9535 & 0.99 & $(0.66 ; 1.49)$ \\
\hline miR screening & -0.478 & 0.2051 & 0.0226 & 0.62 & $(0.41 ; 0.93)$ \\
\hline Sample type & -0.415 & 0.1361 & 0.0032 & 0.66 & $(0.50 ; 0.87)$ \\
\hline Single/multiple & 0.254 & 0.2299 & 0.2728 & 1.29 & $(0.82 ; 2.04)$ \\
\hline
\end{tabular}

Tau-squared estimate $=0.2689$ (convergence is achieved after 6 iterations). Restricted Maximum Likelihood estimation (REML). No. studies $=48$ containing 77 miRNAs. Filter OFF. Add 1/2 to all cells of the studies with zero. Cte, constant coefficient; S, statistic; RDOR, relative diagnostic odds ratio.

B, Four covariates

\begin{tabular}{|c|c|c|c|c|c|}
\hline Variables & Coeff. & Std. Err & P-value & RDOR & $(95 \% \mathrm{CI})$ \\
\hline Cte. & 3.048 & 0.4183 & 0.0000 & - & - \\
\hline $\mathrm{S}$ & -0.300 & 0.0730 & 0.0001 & - & - \\
\hline TNM stage & 0.188 & 0.1078 & 0.0851 & 1.21 & $(0.97 ; 1.50)$ \\
\hline miR screening & -0.479 & 0.2029 & 0.0209 & 0.62 & $(0.41 ; 0.93)$ \\
\hline Sample type & -0.416 & 0.1329 & 0.0025 & 0.66 & $(0.51 ; 0.86)$ \\
\hline Single/multiple & 0.255 & 0.2272 & 0.2658 & 1.29 & $(0.82 ; 2.03)$ \\
\hline
\end{tabular}

Tau-squared estimate $=0.2606$ (convergence is achieved after 6 iterations). Restricted Maximum Likelihood estimation (REML). No. studies $=48$ containing 77 miRNAs. Filter OFF. Add $1 / 2$ to all cells of the studies with zero. Cte, constant coefficient; S, Statistic; RDOR, relative diagnostic odds ratio.

C, Three covariates

\begin{tabular}{|c|c|c|c|c|c|}
\hline Variables & Coeff. & Std. Err & P-value & RDOR & $(95 \% \mathrm{CI})$ \\
\hline Cte. & 3.364 & 0.3156 & 0.0000 & - & - \\
\hline $\mathrm{S}$ & -0.328 & 0.0696 & 0.0000 & - & - \\
\hline TNM stage & 0.176 & 0.1086 & 0.1085 & 1.19 & $(0.96 ; 1.48)$ \\
\hline miR screening & -0.402 & 0.1932 & 0.0409 & 0.67 & $(0.46 ; 0.98)$ \\
\hline Sample type & -0.428 & 0.1340 & 0.0021 & 0.65 & $(0.50 ; 0.85)$ \\
\hline
\end{tabular}

Tau-squared estimate $=0.2727$ (convergence is achieved after 6 iterations). Restricted Maximum Likelihood estimation (REML). No. studies $=48$ containing 77 miRNAs. Filter OFF. Add 1/2 to all cells of the studies with zero. Cte, constant coefficient; S, Statistic; RDOR, relative diagnostic odds ratio.

D, Two covariates

\begin{tabular}{|c|c|c|c|c|c|}
\hline Variables & Coeff. & Std. Err & P-value & RDOR & $(95 \% \mathrm{CI})$ \\
\hline Cte. & 3.630 & 0.2759 & 0.0000 & - & - \\
\hline S & -0.328 & 0.0706 & 0.0000 & - & - \\
\hline miR screening & -0.355 & 0.1944 & 0.0716 & 0.70 & $(0.48 ; 1.03)$ \\
\hline Sample type & -0.476 & 0.1328 & 0.0006 & 0.62 & $(0.48 ; 0.81)$ \\
\hline
\end{tabular}

Tau-squared estimate $=0.2899$ (convergence is achieved after 6 iterations). Restricted Maximum Likelihood estimation (REML). No. studies=48 containing 77 miRNAs. Filter OFF. Add 1/2 to all cells of the studies with zero. Cte, constant coefficient; S, Statistic; RDOR, relative diagnostic odds ratio. 
Table III. Continued.

E, One covariate

\begin{tabular}{lccccc}
\hline Variables & Coeff. & Std. Err & P-value & RDOR & $(95 \%$ CI $)$ \\
\hline Cte. & 3.474 & 0.2661 & 0.0000 & - & - \\
S & -0.335 & 0.0717 & 0.0000 & - & - \\
Sample type & -0.445 & 0.1340 & 0.0014 & 0.64 & $(0.49 ; 0.84)$ \\
\hline
\end{tabular}

Tau-squared estimate $=0.3116$ (convergence is achieved after 6 iterations). Restricted Maximum Likelihood estimation (REML). No. studies $=48$ containing 77 miRNAs. Filter OFF. Add 1/2 to all cells of the studies with zero. Cte, constant coefficient; S, Statistic; RDOR, relative diagnostic odds ratio.
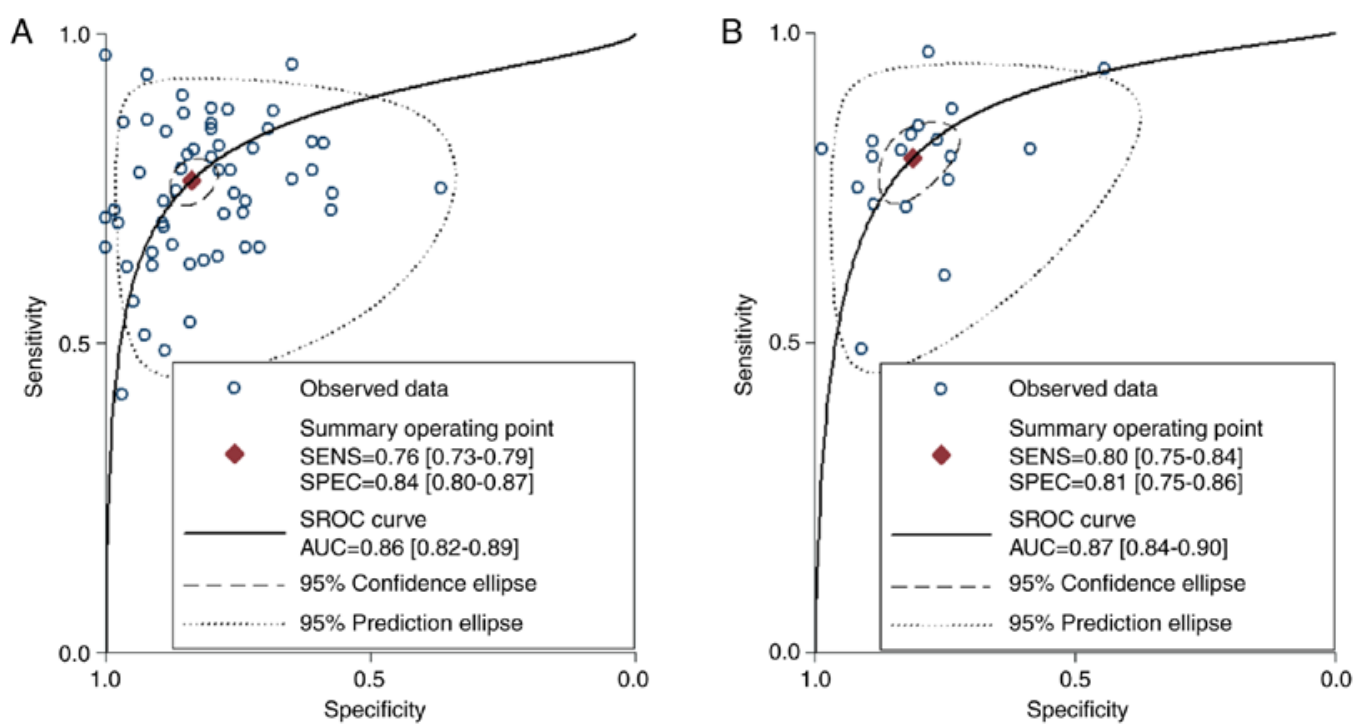

Figure 8. Summary of AUROC of circulating miRNAs from (A) upregulated miRNAs and (B) downregulated miRNAs for the diagnosis of gastric cancer patients.

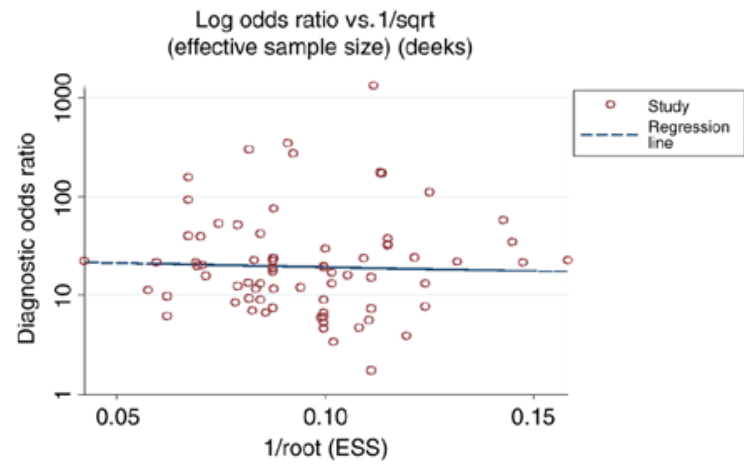

Figure 9. Deek's funnel plots used to estimate publication bias for discrimination of miRNAs in patients with GC. No evidence of publication bias was explored.

results, the study sample type could be considered as a source of heterogeneity for GC detection in meta-regression.

Publication bias. Deeks' funnel plot asymmetry test was applied to explore the publication bias of meta-analysis in diagnostic accuracy (24). The slope coefficient was associated with a P-value of 0.756 for GC detection (Fig. 9), suggesting a low likelihood of publication bias in our meta-analysis.

\section{Discussion}

GC is responsible for the highest number of cancer-related mortalities (74), and the egregious mortality of GC is immeasurably more acute than ever before, primarily since the majority of patients have a terminal disease at stage III or IV at the time of diagnosis (75). In addition, there are many investigated biomarkers, such as CEA and CA724, which lack sufficient sensitivity and specificity for early GC diagnosis (76), and universal screening tools, such as endoscopic examinations and biopsies, are invasive, unpleasant, and inconvenient, leading to potential errors in GC detection. Hence, ideal non-invasive biomarkers are urgently required to reinforce GC detection. At present, a large number of studies on the search for novel tumor biomarkers have revealed that miRNAs may play a pivotal role in cancer suppression, owing to the diverse miRNA expression levels that are observed between cancer patients and healthy controls $(45,48,77,78)$. Subsequently, research has gravitated towards miRNAs as biological markers for tumor diagnosis. Nevertheless, the results of research on the use of miRNAs for gastrointestinal cancer detection are conflicting among different studies $(33,38,44,45,47,48,79,80)$. To the best of our knowledge, several meta-analysis studies have been 
undertaken to determine the differentially expressed miRNAs in GC patients. Unfortunately, as a result of insufficient data or inconsistent results, abundant heterogeneity influenced the results. Furthermore, the accuracy of performance using the pooled results influenced interpretation. In the present meta-analysis, we included 48 studies involving miRNA expression profiling to systematically and comprehensively evaluate the potential diagnostic value of circulating miRNAs as diagnostic markers for GC. We considered different perspectives while avoiding statistical outcomes that included the absence of homogeneity.

The pooled outcomes of sensitivity, specificity, and AUC $(0.76,0.81$, and 0.86 , respectively) with the random effects model revealed that circulating miRNAs have better diagnostic value than CEA and CAA199 (AUC of 0.55 and 0.60, respectively) in distinguishing GC patients from control groups. Moreover, the DOR of circulating miRNAs for GC detection was 15.72, reflecting higher diagnostic performance as a combinative parameter of sensitivity and specificity. In fact, by pooling data in this manner, the diagnostic value would be inaccurate due to the significant heterogeneity and diagnostic threshold. Thus, we could not interpret the statistical outcomes blindly while neglecting high heterogeneity. Additionally, it was suitable to explore subgroup and regression analyses.

From the subgroup analysis, our results indicated that the non-microarray screening approach, multiple miRNA assay, and serum-based miRNA assay manifested a relatively higher diagnostic value and accuracy for GC than the single-miRNA, microarray profiling screening, and plasma-based miRNA groups. The altered expression of the single miRNA in serum or plasma fluctuated not only in GC but also in other tumors, infectious diseases, nonspecific inflammation, and acute injuries. In other words, single miRNAs lacked specificity in cancer detection. However, for multiple miRNAs with complex molecular mechanisms, such as a competing endogenous RNA (ceRNA) network intersecting at tumorigenesis (e.g., initiation and development of a severe neoplasm), the association may be valuable for early GC detection. Hence, studies highlighting individual cancer-specific miRNAs in serum or plasma usually arrived at unsatisfactory results.

Non-microarray screening and serum-based diagnostic tests yielded better outcomes than microarray screening pathways and plasma-based investigations of GC. However, the origin of source-related differences was still unclear. There are many complex factors that must be accounted for, such as lower homogeneity of included studies and a limited number of samples. Therefore, large-scale investigations and multiple center trials should be undertaken in the future to uncover the underlying mechanism of aberrant expression of miRNAs and to determine whether the source-related discrepancies truly exist or not.

Another finding was that the diagnostic value of miRNAs in early stages I-II and high expression groups were approximately similar to those in stages I-IV and lower expression groups in the detection of GC. In the GC microenvironment, a variety of differentiated tumor cells and cancer-associated cells, such as different types of immune cells and cells with different proliferative activity, lead to the transcriptome dysfunction during the tumorigenesis process due to inactivation of tumor suppressors and activation of proto-oncogenes.
With respect to cancer, immune cells have the capacity to release exosomes that accompany cell migration, shuttling the ceRNA network into circulation (81), whereas the circulating tumor cells may express non-coding RNA under the control of an oncogene. Moreover, the overexpression of miRNAs may silence the mRNAs from tumor suppressor genes and downregulated miRNAs may facilitate the expression of oncogene mRNAs by binding the 3'-untranslated region of the target mRNA. In early and advanced GC, the aberrant and abundant expression of some miRNAs may be associated with this process. Moreover, a lack of specificity in the aforementioned may occur not only in GC but in many tumors, leading to various conclusions regarding $\mathrm{GC}$ discrimination. For instance, Liu et al (50) suggested that miRNA expression levels during earlier stages (I and II) were different from those during later stages (III and IV), exhibiting that this miRNA could be valuable for the early detection of GC. Evidently, we arrived at a paradox with our results conflicting with the conclusions of these authors. Therefore, it is difficult to distinguish which is a suitable and specific biomarker for GC. It would be beneficial to study individual miRNAs by determining their molecular mechanism rather than using the combination methodology.

The present study does have some limitations that must be addressed. First, methodologies for a precise uniform quantification of miRNAs face a lack of consistent criteria, limiting the comparisons made between studies that are conducted by different laboratories who have their own study design, use of miRNA chips, pathology type, localization of GC lesions, and different endogenous miRNA references. Second, there are some specific circulating miRNAs that are always prone to be selected by certain studies in determining the correlation between grade and stage of cancer. Consequently, a standardized protocol, which would be preferable, is required to abate bias. Moreover, the included studies in the present meta-analysis only distinguished the tumor patients from healthy controls, but other risk factors, such as chronic gastritis, infectious disease, genetic, ulcers, and diet, were not included and these may contribute to altered miRNA expression $(4,82)$. Therefore, further accurate studies on the use of miRNAs for distinguishing cancer from other diseases are urgently needed.

In conclusion, our meta-analysis found that the combination of multiple miRNAs, non-microarray chip screening, and serum-based miRNA assays may present a better performance for the diagnosis of GC. However, many unclear molecular mechanisms hindered discovery for clear GC detection biomarkers. Therefore, the results should be interpreted cautiously given the uncertainty of the results. Further large-scale prospective studies are required to validate the potential applicability in human cancer diagnosis.

\section{Acknowledgements}

I would like to thank all authors that were involved in this study. In addition, we are appreciative of the support from Professors YPW and WMS.

\section{Funding}

This study was supported by the National Natural Science Foundation of China (81570783), the National Key Research 
and Development Program of China (2016YFC1302200), the Gansu Natural Science Foundation of China (1606RJZA136), the Health Industry Research Project of the Gansu Province (GWGL2014-01), the Scientific Research Project of Traditional Chinese Medicine Administration in Gansu Province (GZK-2017-48), the Open Fund of State Key Laboratory of Cancer Biology (CBSKL201718), and the Fundamental Research Funds for the Central Universities (lzujbky-2015-258).

\section{Availability of data and materials}

All data generated or analyzed in this study are included in this published article.

\section{Authors' contributions}

HuiW, KP, XGL, BXL, HSZ, HuanW, HaoW, WMS and YPW contributed to the conception and design of the study, literature review and analysis, drafting and critical revision and editing, and approval of the final version.

\section{Ethics approval and consent to participate}

Not applicable.

\section{Patient consent for publication}

Not applicable.

\section{Competing interests}

The authors declare that they have no competing interests.

\section{References}

1. Liu L, Wang S, Cao X and Liu J: Diagnostic value of circulating microRNAs for gastric cancer in Asian populations: A meta-analysis. Tumor Biol 35: 11995-2004, 2014.

2. World Health Organization. Cancer Fact Sheets: Available from: URL: http://globocan.iarc.fr/Pages/fact_sheets_population.aspx.

3. Duguan FU: Epigenetic alterations in gastric cancer (Review). Mol Med Rep 12: 3223-3230, 2015.

4. Wang QX,Zhu YQ,Zhang H and Xiao J: Altered MiRNA expression in gastric cancer: A systematic review and meta-analysis Cell Physiol Biochem 35: 933-944, 2015.

5. Chen W, Zheng R, Baade PD, Zhang S, Zeng H, Bray F, Jemal A, $\mathrm{Yu}$ XQ and He J: Cancer statistics in China, 2015. CA Cancer J Clin 66: 115-132, 2016.

6. Charalampakis N, Economopoulou P, Kotsantis I, Tolia M, Schizas D, Liakakos T, Elimova E, Ajani JA and Psyrri A: Medical management of gastric cancer: A 2017 update. Cancer Med 7: 123-133, 2018.

7. Hu N, Yin JF, Ji Z, Hong Y, Wu P, Bian B, Song Z, Li R, Liu Q and Wu F: Strengthening gastric cancer therapy by trastuzumab-conjugated nanoparticles with simultaneous encapsulation of anti-MiR-21 and 5-Fluorouridine. Cell Physiol Biochem 44: $2158-2173,2017$

8. Huang S, Wang J, Li J, Luo Q, Zhao M, Zheng L, Dong X, Chen C, Che Y, Liu P, et al: Serum microRNA expression profile as a diagnostic panel for gastric cancer. Jpn J Clin Oncol 46: 811, 2016.

9. Kitano S, Shiraishi N, Uyama I Sugihara K and Tanigawa N; Japanese Laparoscopic Surgery Study Group: A multicenter study on oncologic outcome of laparoscopic gastrectomy for early cancer in Japan. Ann Surg 245: 68-72, 2007.

10. Uedo N, Takeuchi Y and Ishihara R: Endoscopic management of early gastric cancer: Endoscopic mucosal resection or endoscopic submucosal dissection: Data from a Japanese high-volume center and literature review. Ann Gastroenterol 25: 281-290, 2012.
11. Kaneko S and Yoshimura T: Time trend analysis of gastric cancer incidence in Japan by histological types, 1975-1989. Br J Cancer 84: 400-405, 2001.

12. Bonenkamp JJ, Hermans J, Sasako M, van de Velde CJ, Welvaart K, Songun I, Meyer S, Plukker JT, Van Elk P, Obertop H, et al: Extended lymph-node dissection for gastric cancer. N Engl J Med 340: 908-914, 1999.

13. Garrido M, Bustos M, Orellana E, Madrid J, Galindo H, Sánchez C, Pimentel F, Guzmán S, Ibáñez L, Butte JM, et al: Postoperative radio chemotherapy in locally advanced gastric cancer. Rev Med Chil 136: 844-850, 2008 (In Spanish).

14. Madhavan D, Cuk K, Burwinkel B and Yang R: Cancer diagnosis and prognosis decoded by blood-based circulating microRNA signatures. Front Genet 4: 116, 2013.

15. Komatsu S, Ichikawa D, Tsujiura M, Konishi H, Takeshita $H$, Nagata H, Kawaguchi T, Hirajima S, Arita T, Shiozaki A, et al: Prognostic impact of circulating miR-21 in the plasma of patients with gastric carcinoma. Anticancer Res 33: 271-276, 2013.

16. Chen CZ: MicroRNAs as oncogenes and tumor suppressors. $\mathrm{N}$ Engl J Med 353: 1768-1771, 2005.

17. Lee RC, Feinbaum RL and Ambros V: The C. elegans heterochronic gene lin-4 encodes small RNAs with antisense complementarity to lin-14. Cell 75: 843-854, 1993.

18. Gorur A, Balci Fidanci S, Dogruer Unal N, Ayaz L, Akbayir S, Yildirim Yaroglu H, Dirlik M, Serin MS and Tamer L: Determination of plasma microRNA for early detection of gastric cancer. Mol Biol Rep 40: 2091-2096, 2013.

19. Mitchell PS, Parkin RK, Kroh EM, Fritz BR, Wyman SK, Pogosova-Agadjanyan EL, Peterson A, Noteboom J, O'Briant KC, Allen A, et al: Circulating microRNAs as stable blood-based markers for cancer detection. Proc Natl Acad Sci USA 105: 10513-10518, 2008.

20. Whiting PF, Rutjes AW, Westwood ME, Mallett S, Deeks JJ, Reitsma JB, Leeflang MM, Sterne JA and Bossuyt PM; QUADAS-2 Group: QUADAS-2: A revised tool for the quality assessment of diagnostic accuracy studies. Ann Intern Med 155: 529-536, 2011.

21. Deeks JJ and Morris JM: 6 Evaluating diagnostic tests. Baillière's Clin Obstet Gynaecol 10: 613-630, 1996.

22. Deeks JJ: Systematic reviews in health care: Systematic reviews of evaluations of diagnostic and screening tests. BMJ 323: 157-162, 2001.

23. Jaeschke R, Guyatt GH, Sackett DL. Users' guides to the medical literature. III. How to use an article about a diagnostic test. B. What are the results and will they help me in caring for my patients? The Evidence-Based Medicine Working Group. JAMA 271: 703-707, 1994.

24. Deeks JJ, Macaskill P and Irwig L: The performance of tests of publication bias and other sample size effects in systematic reviews of diagnostic test accuracy was assessed. J Clin Epidemiol 58: 882-893, 2005.

25. Higgins JP, Thompson SG, Deeks JJ and Altman DG: Measuring inconsistency in meta-analyses. BMJ 327: 557-560, 2003.

26. Jackson D, White IR and Thompson SG: Extending DerSimonian and Laird's methodology to perform multivariate random effects meta-analyses. Stat Med 29: 1282-1297, 2010.

27. Peng WZ, Ma R, Wang F, Yu J and Liu ZB: Role of miR-191/425 cluster in tumorigenesis and diagnosis of gastric cancer. Int J Mol Sci 15: 4031-4048, 2014.

28. Qiu X, Zhang J, Shi W, Liu S, Kang M, Chu H, Wu D, Tong N, Gong W, Tao G, et al: Circulating microRNA-26a in plasma and its potential diagnostic value in gastric cancer. PLoS One 11: e0151345, 2016.

29. Tsujiura M, Komatsu S, Ichikawa D, Shiozaki A, Konishi H, Takeshita H, Moriumura R, Nagata H, Kawaguchi T, Hirajima S, et al: Circulating miR-18a in plasma contributes to cancer detection and monitoring in patients with gastric cancer. Gastric Cancer 18: 271-279, 2015.

30. Zhang J, Song Y, Zhang C, Zhi X, Fu H, Ma Y, Chen Y, Pan F, Wang K, Ni J, et al: Circulating MiR-16-5p and MiR-19b-3p as two novel potential biomarkers to indicate progression of gastric cancer. Theranostics 5: 733-745, 2015.

31. Tsai MM, Wang CS, Tsai CY, Huang CG, Lee KF, Huang HW, Lin YH, Chi HC, Kuo LM, Lu PH and Lin KH: Circulating microRNA-196a/b are novel biomarkers associated with metastatic gastric cancer. Eur J Cancer 64: 137-148, 2016.

32. Su ZX, Zhao J, Rong ZH, Wu YG, Geng WM and Qin CK: Diagnostic and prognostic value of circulating miR-18a in the plasma of patients with gastric cancer. Tumor Biol 35: 12119-12125, 2014. 
33. Valladares-Ayerbes M, Reboredo M, Medina-Villaamil V, Iglesias-Díaz P, Lorenzo-Patiño MJ, Haz M, Santamarina I, Blanco M, Fernández-Tajes J, Quindós M, et al: Circulating miR-200c as a diagnostic and prognostic biomarker for gastric cancer. J Transl Med 10: 186, 2012.

34. Zhu X, Yang Z, Zhang J and Liu H: Investigation of microRNA-145 as a serum diagnostic and prognostic biomarker for gastric cancer: A Chinese cohort-based study. Int J Clin Exp Med 10: 9440-9447, 2017.

35. Zhuang K, Han K, Tang H, Yin X, Zhang J, Zhang X and Zhang L: Up-regulation of plasma miR-23b is associated with poor prognosis of gastric cancer. Med Sci Monit 22: 356-361, 2016.

36. Hung PS, Chen CY, Chen WT, Kuo CY, Fang WL, Huang KH, Chiu PC and Lo SS: MiR-376c promotes carcinogenesis and serves as a plasma marker for gastric carcinoma. PLoS One 12: e0177346, 2017.

37. Le Q, Jianhua N, Mulati, Yu X and Jiageng H: Increased miR-25 expression in serum of gastric cancer patients is correlated with CA19-9 and acts as a potential diagnostic biomarker. Open Medicine 12: 266-270, 2017.

38. Li BS, Zhao YL, Guo G, Li W, Zhu ED, Luo X, Mao XH, Zou QM, Yu PW, Zuo QF, et al: Plasma microRNAs, miR-223, miR-21 and miR-218, as novel potential biomarkers for gastric cancer detection. PLoS One 7: e41629, 2012.

39. Li F, Guo Y, Liu J and Zhang R: The significance of elevated plasma expression of microRNA $106 \mathrm{~b} \sim 25$ clusters in gastric cancer. PLoS One 12: e0178427, 2017.

40. Li M, Gu K, Liu W, Xie X and Huang X: MicroRNA-200c as a prognostic and sensitivity marker for platinum chemotherapy in advanced gastric cancer. Oncotarget 8: 51190-51199, 2017.

41. Hou X, Zhang M and Qiao H: Diagnostic significance of miR-106a in gastric cancer. Int J Clin Exp Pathol 8: 13096-13101, 2015.

42. Hou CG, Luo XY and Li G: Diagnostic and prognostic value of serum MicroRNA-206 in patients with gastric cancer. Cell Physiol Biochem 39: 1512-1520, 2016.

43. Zhang WH, Gui JH, Wang CZ, Chang Q, Xu SP, Cai CH, Li YN, Tian YP, Yan L and Wu B: The identification of miR-375 as a potential biomarker in distal gastric adenocarcinoma. Oncol Res 20: 139-147, 2012

44. Song MY, Pan KF, Su HJ, Zhang L, Ma JL, Li JY, Yuasa Y, Kang D, Kim YS and You WC: Identification of serum microRNAs as novel non-invasive biomarkers for early detection of gastric cancer. PLoS One 7: e33608, 2012.

45. Tsujiura M, Ichikawa D, Komatsu S, Shiozaki A, Takeshita H, Kosuga T, Konishi H, Morimura R, Deguchi K, Fujiwara H, et al: Circulating microRNAs in plasma of patients with gastric cancers. Br J Cancer 102: 1174-1179, 2010.

46. Wang B and Zhang Q: The expression and clinical significance of circulating microRNA-21 in serum of five solid tumors. J Cancer Res Clin Oncol 138: 1659-1666, 2012.

47. Zhu C, Ren C, Han J, Ding Y, Du J, Dai N, Dai J, Ma H, Hu Z, Shen $\mathrm{H}$, et al: A five-microRNA panel in plasma was identified as potential biomarker for early detection of gastric cancer. $\mathrm{Br}$ J Cancer 110: 2291-2299, 2014.

48. Liu R, Zhang C, Hu Z, Li G, Wang C, Yang C, Huang D, Chen X, Zhang $\mathrm{H}$, Zhuang R, et al: A five-microRNA signature identified from genome-wide serum microRNA expression profiling serves as a fingerprint for gastric cancer diagnosis. Eur J Cancer 47 784-791, 2011.

49. Konishi H, Ichikawa D, Komatsu S, Shiozaki A, Tsujiura M, Takeshita H, Morimura R, Nagata H, Arita T, Kawaguchi T, et al: Detection of gastric cancer-associated microRNAs on microRNA microarray comparing pre- and post-operative plasma. $\mathrm{Br}$ J Cancer 106: 740-747, 2012.

50. Liu H, Zhu L, Liu B, Yang L, Meng X, Zhang W, Ma Y and Xiao H: Genome-wide microRNA profiles identify miR-378 as a serum biomarker for early detection of gastric cancer. Cancer Lett 316: 196-203, 2012

51. Liu X, Kwong A, Sihoe A and Chu KM: Plasma miR-940 may serve as a novel biomarker for gastric cancer. Tumor Biol 37: $3589-3597,2016$

52. Hu Y, Wang J, Han Y, Liu Q and Niu Q: Serum miR-133a is down-regulated and associated with the diagnosis of patients with gastric cancer. Int J Clin Exp Pathol 9: 2015-2020, 2016

53. Sun Y, Ma J, Hu M, Zheng X, Li J and Gu Wei: Level of miR-183 in peripheral blood of the patients with gastric cancer. J Shanghai Jiaotong Univ 37: 75-79, 2017.
54. Li B and Zhang H: Plasma microRNA-320 is a potential diagnostic and prognostic bio-marker in gastric cancer. Int J Clin Exp Pathol 10: 7356-7361, 2017.

55. Wu J, Li G, Yao Y, Wang Z, Sun W and Wang J: MicroRNA-421 is a new potential diagnosis biomarker with higher sensitivity and specificity than carcinoembryonic antigen and cancer antigen 125 in gastric cancer. Biomarkers 20: 58-63, 2015.

56. Zeng Q, Jin C, Chen W, Xia F, Wang Q, Fan F, Du J, Guo Y, Lin C, Yang K, et al: Downregulation of serum miR-17 and miR-106b levels in gastric cancer and benign gastric diseases. Chin J Cancer Res 26: 711-716, 2014.

57. Zhou H, Xiao B, Zhou F, Deng H, Zhang X, Lou Y, Gong Z, Du C and Guo J: MiR-421 is a functional marker of circulating tumor cells in gastric cancer patients. Biomarkers 17: 104-110, 2012.

58. Zhou X, Zhu W, Li H, Wen W, Cheng W, Wang F, Wu Y, Qi L, Fan Y, Chen Y, et al: Diagnostic value of a plasma microRNA signature in gastric cancer: A microRNA expression analysis. Sci Rep 5: 11251, 2015

59. Wu J, Li G, Wang Z, Yao Y, Chen R, Pu X and Wang J: Circulating MicroRNA-21 is a potential diagnostic biomarker in gastric cancer. Dis Markers 2015: 435656, 2015.

60. Wu D, Cao G, Huang Z, Jin K, Hu H, Yu J and Zeng Y: Decreased miR-503 expression in gastric cancer is inversely correlated with serum carcinoembryonic antigen and acts as a potential prognostic and diagnostic biomarker. OncoTargets Ther 10: 129-135, 2017.

61. Wang H, Wang L, Wu Z, Sun R, Jin H, Ma J, Liu L, Ling R, Yi J, Wang L, et al: Three dysregulated microRNAs in serum as novel biomarkers for gastric cancer screening. Med Oncol 31: 298, 2014.

62. Shin VY, Ng EK, Chan VW, Kwong A and Chu KM: A three-miRNA signature as promising non-invasive diagnostic marker for gastric cancer. Mol Cancer 14: 202, 2015.

63. Cui YJ, Xie XH, Xing YF, Yuan ZH, Wu QY and Wei YM: Feasibility study on plasmatic microRNA-27b-3p as a potential biomarker for early diagnosis of gastric cancer. Tumor 35 : 183-189, 2015.

64. Fu Z, Qian F, Yang X, Jiang H, Chen Y and Liu S: Circulating miR-222 in plasma and its potential diagnostic and prognostic value in gastric cancer. Med Oncol 31: 164, 2014.

65. Liu H, Yang Z, Zhang J and Zhu X: MicroRNA-217 in plasma: A potential biomarker in gastric cancer. Int J Clin Exp Med 10: 3313-3320, 2017

66. Zhou H, Guo JM, Lou YR, Zhang XJ, Zhong FD, Jiang Z, Cheng J and Xiao BX: Detection of circulating tumor cells in peripheral blood from patients with gastric cancer using microRNA as a marker. J Mol Med (Berl) 88: 709-717, 2010.

67. Zhou X and Zhang G: Exosome-mediated transfer of miR-223 increases tumor malignancy in gastric cancer. J Gastroen Hepatol (Australia) 31: 45-46, 2016.

68. Zheng Y, Cui L, Sun W, Zhou H, Yuan X, Huo M, Chen J, Lou Y and Guo J: MicroRNA-21 is a new marker of circulating tumor cells in gastric cancer patients. Cancer Biomark 10: 71-77, 2011.

69. Li H, Wu Q, Li T, Liu C, Xue L, Ding J, Shi Y and Fan D: The miR-17-92 cluster as a potential biomarker for the early diagnosis of gastric cancer: Evidence and literature review. Oncotarget 8: 45060-45071, 2017.

70. Park JL, Kim M, Song KS, Kim SY and Kim YS: Cell-Free miR-27a, a potential diagnostic and prognostic biomarker for gastric cancer. Genomics Inform 13: 70-75, 2015.

71. Li C, Li JF, Cai Q, Qiu QQ, Yan M, Liu BY and Zhu ZG: MiRNA-199a-3p: A potential circulating diagnostic biomarker for early gastric cancer. J Surg Oncol 108: 89-92, 2013.

72. Li C, Li JF, Cai Q, Qiu QQ, Yan M, Liu BY and Zhu ZG: MiRNA-199a-3p in plasma as a potential diagnostic biomarker for gastric cancer. Ann Surg Oncol 20 (Suppl 3): S397-S405, 2013 .

73. Liu S, Suo J, Wang C, Sun X, Wang D, He L, Zhang Y and Li W: Prognostic significance of low miR-144 expression in gastric cancer. Cancer Biomark 20: 547-552, 2017.

74. Jemal A, Bray F, Center MM, Ferlay J, Ward E and Forman D: Global cancer statistics. CA Cancer J Clin 61: 69-90, 2011.

75. Lurje G, Schiesser M, Claudius A and Schneider PM: Circulating tumor cells in gastrointestinal malignancies: Current techniques and clinical implications. J Oncol 2010: 392652, 2010.

76. Leung WK, Wu MS, Kakugawa Y, Kim JJ, Yeoh KG, Goh KL, Wu KC, Wu DC, Sollano J, Kachintorn U, et al: Screening for gastric cancer in Asia: Current evidence and practice. Lancet Oncol 9: 279-287, 2008. 
77. Redova M, Sana $\mathbf{J}$ and Slaby O: Circulating miRNAs as new blood-based biomarkers for solid cancers. Future Oncol 9: 387-402, 2013

78. Shen J, Stass SA and Jiang F: MicroRNAs as potential biomarkers in human solid tumors. Cancer Lett 329: 125-136, 2013.

79. Ng EK, Chong WW, Jin H, Lam EK, Shin VY, Yu J, Poon TC, Ng SS and Sung JJ: Differential expression of microRNAs in plasma of patients with colorectal cancer: A potential marker for colorectal cancer screening. Gut 58: 1375-1381, 2009.

80. Zanutto S, Pizzamiglio S, Ghilotti M, Bertan C, Ravagnani F Perrone F, Leo E, Pilotti S, Verderio P, Gariboldi $M$ and Pierotti MA: Circulating miR-378 in plasma: A reliable haemolysis independent biomarker for colorectal cancer. $\mathrm{Br}$ J Cancer 110: 1001-1007, 2014.
81. Valadi H, Ekström K, Bossios A, Sjöstrand M, Lee JJ and Lötvall JO: Exosome-mediated transfer of mRNAs and microRNAs is a novel mechanism of genetic exchange between cells. Nat Cell Biol 9: 654-659, 2007.

82. Matsushima $\mathrm{K}$, Isomoto $\mathrm{H}$, Inoue $\mathrm{N}$, Nakayama $\mathrm{T}$, Hayashi $\mathrm{T}$, Nakayama M, Nakao K, Hirayama T and Kohno S: MicroRNA signatures in Helicobacter pylori-infected gastric mucosa. Int J Cancer 128: 361-370, 2011.

(i) $(9)$ This work is licensed under a Creative Commons Attribution-NonCommercial-NoDerivatives 4.0 International (CC BY-NC-ND 4.0) License. 